\title{
On the Potential and Limits of Large Area Seeding for Photovoltaic Silicon
}

\author{
Nathan Stoddard ${ }^{1}$, Bianca Gründig-Wendrock ${ }^{2}$, Andreas Krause ${ }^{2}$, Daniel Oriwol ${ }^{2}$, Mariana Bertoni ${ }^{3}$, Tine \\ Uberg Naerland ${ }^{3}$, lan Witting ${ }^{4}$ and Lamine Sylla ${ }^{2}$ \\ 1 SolarWorld Americas, Hillsboro, Oregon, USA \\ 2 SolarWorld Innovations, Freiberg, Germany \\ 3 Dept. of Electrical, Computer and Energy Engineering, Arizona State University, Tempe, \\ Arizona, USA \\ 4 Dept. of Materials Science and Engineering, Northwestern University, Chicago, Illinois, USA
}

Corresponding author info:

Address: 25300 NW Evergreen Road, Hillsboro, OR 97124

Email: nathan.stoddard@solarworld.com

\begin{abstract}
Single crystal production of silicon for solar cell substrates has relied on the Dash neck technique developed more than 50 years ago. The technique is simple and repeatable and enables truly dislocation free crystal growth. It does have drawbacks, however, including limits on throughput and some structural difficulties. It has long been assumed that dislocation-free growth is not possible by any other method. In the 'quasi-mono' crystal growth technique, one of the key elements is the use of large area single crystal seeds. By melting the seeds at near-equilibrium conditions, it is feasible to avoid the production of dislocations during melting. We will review the dislocation relevant details of the large area seeding process and present best case results for dislocation density, including measured minority carrier lifetimes in excess of $1 \mathrm{~ms}$ on p-type material. We will focus on dislocation density exclusive of seed boundaries, but we will also present a potential best-case limit for the technique.
\end{abstract}

Keywords: A1. Directional Solidification; B2. Semiconducting Silicon; B3. Solar Cells; A2. Seed Crystals

\section{Introduction}

Single crystals of silicon, germanium and other materials have been grown in dislocation-free boules for decades using the Czochralski ( $\mathrm{CZ}$ ) and Float Zone (FZ) techniques. The Dash Neck process of pulling a long thin section and then expanding the crystal to a body dimension has been a remarkably successful process[1]. Dislocation-free crystals are valued for their high uniformity, e.g. for semiconductor applications where every square millimeter counts. They also possess low residual stress, even in high pull rate situations, as well as high tensile strength and generally high minority carrier lifetime[2]. The need for a Dash Neck in the crystal growth process is based on the introduction of dislocations from 'thermal shock' at the point when the solid seed is dipped into the melt. This shock is due to the extremely poor pre-heating of the seed in both the Cz and FZ methods. Given the lack of a dedicated heater for the pre-heating of the seed, the seed is primarily heated by radiation from the liquid silicon. 
Because of the large mismatch in emissivity between solid silicon ( 0.70) [3] and liquid silicon ( 0.25) [4], a seed placed near a melt will reach radiative equilibrium at a maximum temperature of

$$
T_{\mathrm{s}}=T_{1} *\left(\varepsilon_{1} / \varepsilon_{\mathrm{s}}\right)^{1 / 4}=1737 \mathrm{~K} *(0.25 / 0.7)^{1 / 4}=1343 \mathrm{~K}
$$

giving a $\Delta T$ of $394 \mathrm{~K}$. The situation is quite different in large area seeding methods, however. Generally, the seed crystal in large area seeding is heated to a temperature very near the melting temperature at quasi-equilibrium thermal conditions before it is contacted with liquid. As a result, thermal shock is not a given assuming low temperature gradients within the large seed and it is at least theoretically possible to achieve dislocation-free seeding without a necking process. Furthermore, the conditions demanding dislocation-free crystals in semiconductor device applications are not present in photovoltaic (PV) applications, where up to $\sim 10^{4}$ dislocations $/ \mathrm{cm}^{2}$ can still deliver high minority carrier lifetime [5]. For this reason, the subject of large-area seeding, as practiced in the Heat Exchanger Method (HEM), Quasimono method (QM), and Kyropolous method, has been of high interest in silicon photovoltaics $[6,7,8]$. In this paper, we will review the sources of dislocations in different large area seeding techniques and present new results of an idealized large-area seeding experiment pointing to the possibilities of uniform, high performance PV substrates.

\section{Methods}

Silicon ingots have been grown in directional solidification furnaces. Feedstock silicon is placed on top of seed crystals and then melted, together with some of the seed crystal. Heat is then extracted through the seed to solidify the crystal. Both typical slip-cast crucibles and high purity quartz have been used as contact materials for the liquid silicon. Dislocations and defects have been quantified by selective etching and scanning microscopy, while minority carrier lifetime has been measured using a Sinton lifetime tool in Transient mode, both for bulk samples and on passivated wafers. White-beam $\mathrm{x}$ ray topography has been performed using a synchrotron radiation source.

\section{Results}

A number of process-induced defects threaten the quality of the seed material and the ingot during large-area seeded crystal growth. First, the seed is typically placed under a significant mass of material in the HEM and QM methods. Local stresses can exceed the threshold to produce dislocations in several ways, see Fig. 1. First, the load may be unevenly supported on the seeds, with individual support points from feedstock contact causing high local stress. Second, the seed may be supported unevenly, either due to small chips, coating or dust particles under the seed, or due to gross undulations in the seed support surface. In either case, the effect is large stresses on the seeds. With larger seed crystals, cracking of the seed material is even possible. Any dislocations generated during the heat-up and meltin process will then propagate into the grown crystal. 
A second major issue specific to the QM growth method is the misalignment of seeds. Even seeds cut with high precision rectilinearity from the same seed crystal can experience misalignment in two ways, see Fig. 2 . There may be an in-plane rotation between seeds, $\phi$, caused by poor packing or a particle between the seeds, and there may be out of plane deflection, $\theta$. Out of plane deflections can arise from variations in the crucible bottom, from seeds placed on the corner radius at the edges, or from small particles underneath them. In any case, a few tenths of a degree misalignment is sufficient to start a dislocation cascade and sub-grain boundary formation.

Another factor affecting seed alignment is an effect where liquid can seep down the sides of the crucible and infiltrate under very small gaps between the seed and the crucible. When the liquid freezes in the cavity, it expands and creates high stress levels, extended defects and seed misorientation.

The effects of some of these process defects can be seen in Fig. 3 and have been reported in [9] for labscale ingots $A$ sample has been cut from the bottom of the crystal at the joint of two seed plates. There was originally a small physical gap between the two seed plates, but most of it has been filled by liquid that flowed down into it and crystallized. The only remaining physical gap is at point (A). A small pinning point (B) between the seed plates has caused an intense local dislocation field. Apart from this, a cellular dislocation structure can be seen throughout the seed, but is especially well defined at (D). The pinning point is also a possible cause for the seed misalignment that is evidenced by the white line gap (C), which is not due to physical space between the seeds (as at the very bottom), but instead from different $x$-ray deflection angles from the two seed crystals. Above the initial solid/liquid interface, the grown crystal quickly devolves into a tangle of dislocations (F). Finally, silicon nitride particles coming from the release coating sprayed on the inner surface of the crucible have settled to the bottom of the melt and become trapped at the transition from melting to crystal growth. These particles are small and do not necessarily cause dislocation tangles like the seed boundaries do.

There are two other transitions worth discussing at the seed/grown crystal interface. First, the seed retains the high oxygen concentrations of its $\mathrm{Cz}$ origins $\left(8-12 \times 10^{17}\right.$ atoms $\left./ \mathrm{cm}^{3}\right)$, but the grown crystal has significantly lower oxygen concentration. Second, it is typically observed in QM ingots that the bottom of the ingot has a larger low-lifetime 'red zone' at the bottom of the ingot than normal multicrystalline ingots[10]. In order to understand this, we conducted an experiment where we use a very thick seed $(>8 \mathrm{~cm})$, large enough that we can maintain high lifetime material in the center despite the in-diffusion of impurities from the crucible. We melted slightly into the seed with the silicon melt above and then started growth. Deep Level Transient Spectroscopy was used to measure iron within $3 \mathrm{~cm}$ on either side of the seed/growth interface. This data was then compared with a one dimensional model of a plane source of impurities allowed to diffuse during a time and temperature profile typical for that part of the ingot in the crystal growth, annealing and cooling phases. The result gives a good match, as shown in Fig. 4. Note that the upper plot is in $\mathrm{mm}$ while the lower is in $\mathrm{cm}$. The FWHM is slightly larger in the measured case, indicating that there may be some enhanced diffusion in the actual process due to structural defects, or that the source layer has a measurable thickness of a few millimeters. The explanation for the iron rich layer could be one of two things. First, it may be that a thin layer of iron-rich liquid is frozen first, before the normal segregating behavior sets up for the impurities. This would involve a non-equilibrium solidification occurring at the very low growth rates of the beginning of growth. A second possibility is that the hottest solid (next to the interface) will have 
the highest solubility for impurities, and may form a source for the impurity layer, but the temperature gradient in the solid would not account for the sharpness of the source.

Many of the drawbacks and limitations identified so far are the results of very practical limitations inherent to production operations: the flatness of the seed support, the precision of seed placement, the impurity levels present in industrial-scale crucibles and coatings, and the stress concentration from heavy feedstock loads. Several of these trace their origin to a process that was developed to leverage furnaces and crucibles developed for the multicrystalline process. In order to investigate the more intrinsic limitations of large-area seeding, we grew several ingots where we eliminated the more practical limitations. High purity materials were used, seeds were placed on a machined-flat surface and feedstock loads were kept sub-critical. In Fig. 5, a photo shows a cross-section of a first ingot, with preferential etching results typical of three areas.

Within the seed itself, the stresses have been minimized to the point where the seed is nearly dislocation-free. At the interface between the seed and the grown material, a low density of dislocations $\left(10^{2}-10^{3} / \mathrm{cm}^{2}\right)$ and twin boundaries can be found. Higher up in the ingot, twin boundaries become more frequent, some of them decorated with dislocations. At the very top, the last to freeze area still has a relatively high dislocation density $\left(>10^{5} / \mathrm{cm}^{2}\right)$. In Fig. 6, a higher density of dislocations $\left(\sim 10^{4} / \mathrm{cm}^{2}\right)$ is found in both the seed and the grown material, as seen in a stitched image of microscope captures. The dislocation density peaks at the seed/growth interface and then decreases further up in the grown material. The photoluminescence scan of the area shows a distinct transition between the seed and the grown material, indicating a minority carrier lifetime transition that is not characterized by an initial solidification of high metal content liquid. It should be noted that the left-right PL changes are due to non-flatness of the sample. The PL contrast is attributed to a change from the high oxygen seed to the low oxygen grown material. The bulk grown material from these ingots has measured as high as 530 microseconds in transient mode measurements on a Sinton lifetime tool, while wafers that were phosphorus-diffused, etched back and passivated with amorphous silicon have measured as high as $1.7 \mathrm{~ms}$ on the $\mathrm{p}$-type material in areas free of dislocation clusters, while areas with dislocation clusters have measured up to 480 microseconds.

\section{Conclusion}

The limiting factors in the quasi-mono implementation of large-area seeded silicon crystallization have been traced to practical difficulties in the industrial setting. Problems such as dislocations, low lifetime and even fractures in the seed crystals arise from an environment that is not optimized for high quality crystal growth. In model experiments where these restrictions have been removed, it has been shown that very low dislocation densities and high lifetimes can be achieved from large area seeding, in some ways exceeding the quality possible from boron-doped $\mathrm{Cz}$ silicon. The real challenge comes in translating the higher standards of the model experiments into workable production solutions, but the goal seems worth the challenge. 


\section{Acknowledgements}

The work presented herein was funded in part by the Office of Energy Efficiency and Renewable Energy (EERE), U.S. Department of Energy, under Award Number DE-EE0006806.

\section{Citations}

1 W. C. Dash, J. Appl. Phys. 30 (1959), p. 459

2 H. Siethoff, from Properties of Crystalline Silicon, R. Hull, ed. (1999), p. 123

3 E. Takasuka et al., J. Appl. Phys. 81, (1997), p. 6384

4 S C Jain et al., J. Phys. D: Appl. Phys. 4 (1971), p. 1207

5 C. Donolato, J. Appl. Phys. 84 (1998), p. 5

6 N. Stoddard, B. Wu, I. Witting, M. C. Wagener, Y. Park, G. A. Rozgonyi, R. Clark, Solid State Phenomena, 131-133, (2007), pp. 1-8

7 Y. Miyamura, H. Harada, K. Jiptner, J. Chen, R.R. Prakash, S. Nakano, B. Gao, K. Kakimoto, T. Sekiguchi, J. Crystal Growth 401 (2014) p. 133

8 K. Nakajima, R. Murai, S. Ono, K. Morishita, M. Kivambe, D. M. Powell, and T. Buonassisi, Japanese Journal of Applied Physics, 54(1), (2015), p. 015504.

9 M.Trempa, C. Reimann, J. Friedrich, G. Müller, A. Krause, L. Sylla, T. Richter, J. Crystal Growth 405 (2014) p. 131

10 M. Trempa, C. Reimann, J. Friedrich, G. Müller, L. Sylla, A. Krause, T. Richter, J. Crystal Growth 429 (2015) p. 56 


\section{Figures}

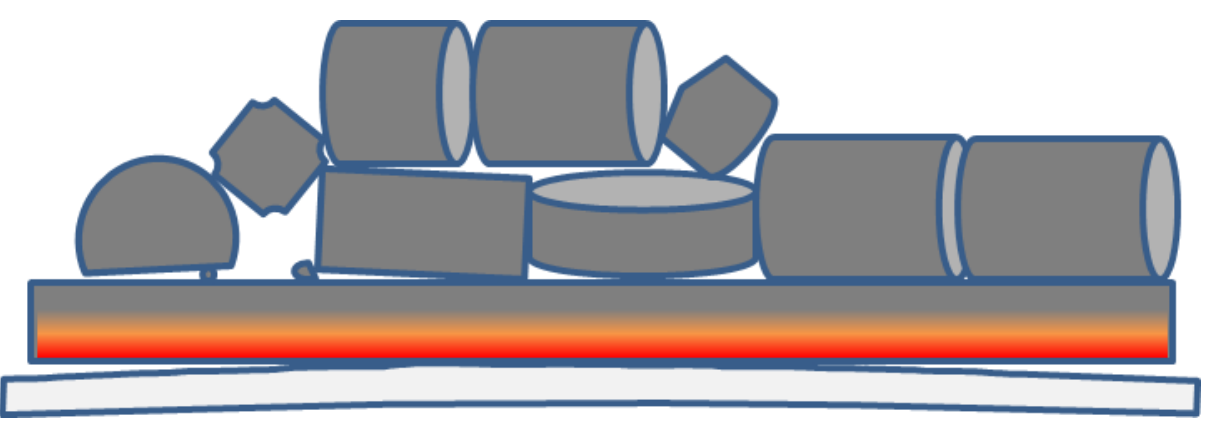

Fig. 1: Large area seed placed on top of an uneven crucible with feedstock point loads applying locally super-critical pressures

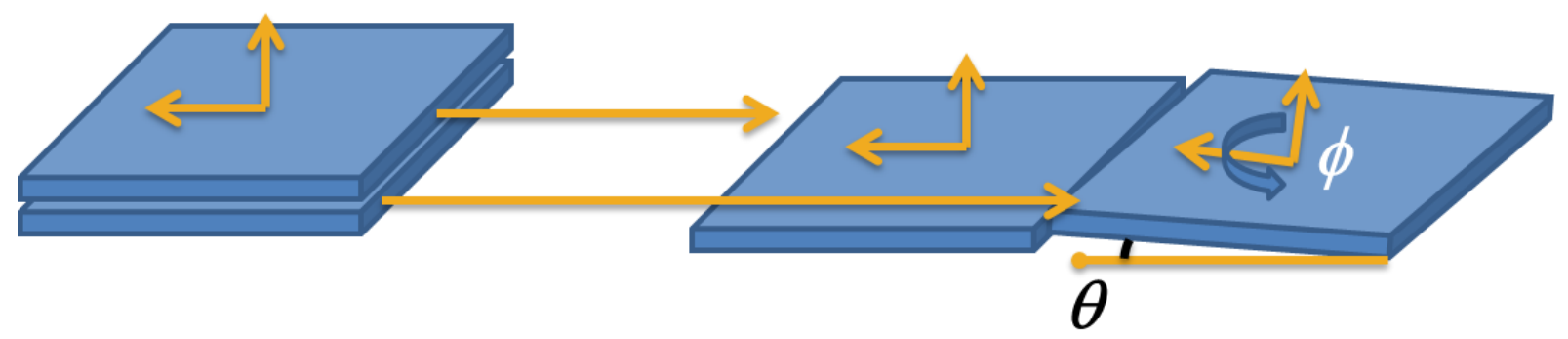

Fig. 2: Seed plates cut from the same block of seed material can still end up with angular deviations due to poor placement or small chips or variations in the supporting surface. 


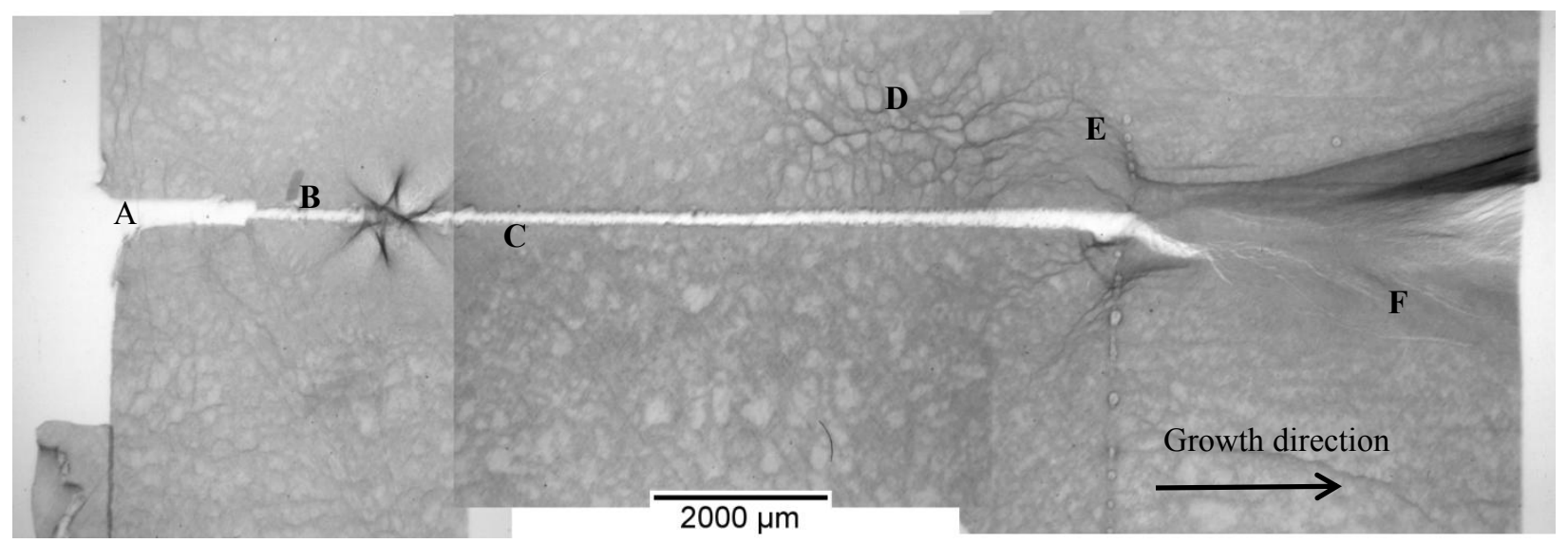

Fig. 3: White Beam X-Ray Topograph of a cross-section of a quasi-mono ingot. A) physical gap between seeds, $B$ ) Binding point with high stress, C) seed alignment gap from $x$-ray deflection, D) cellular dislocation structure, E) $\mathrm{Si}_{3} \mathrm{~N}_{4}$ particles at the seed/growth interface and F) dislocation cascade developing from seed boundary.

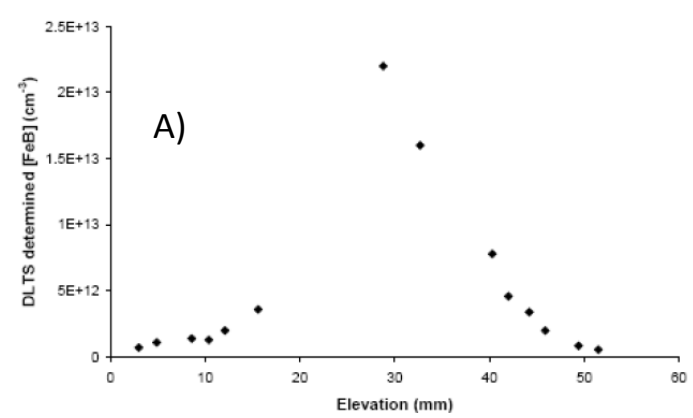

Thin Film Solution

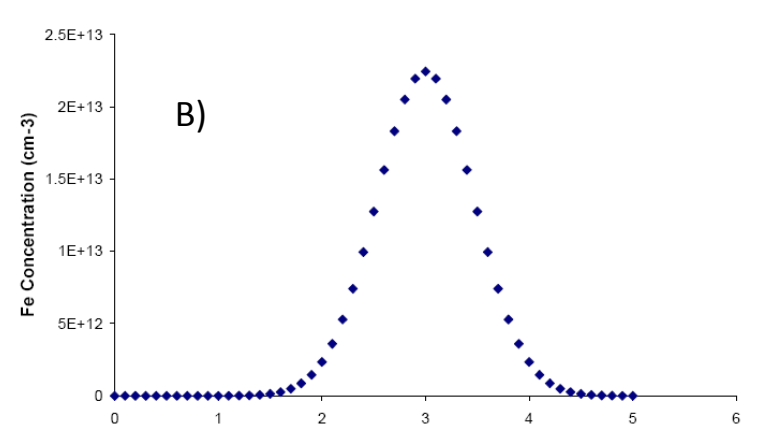

Fig. 4: Comparison between A) measured and B) simulated iron concentrations in a $Q M$ ingot 


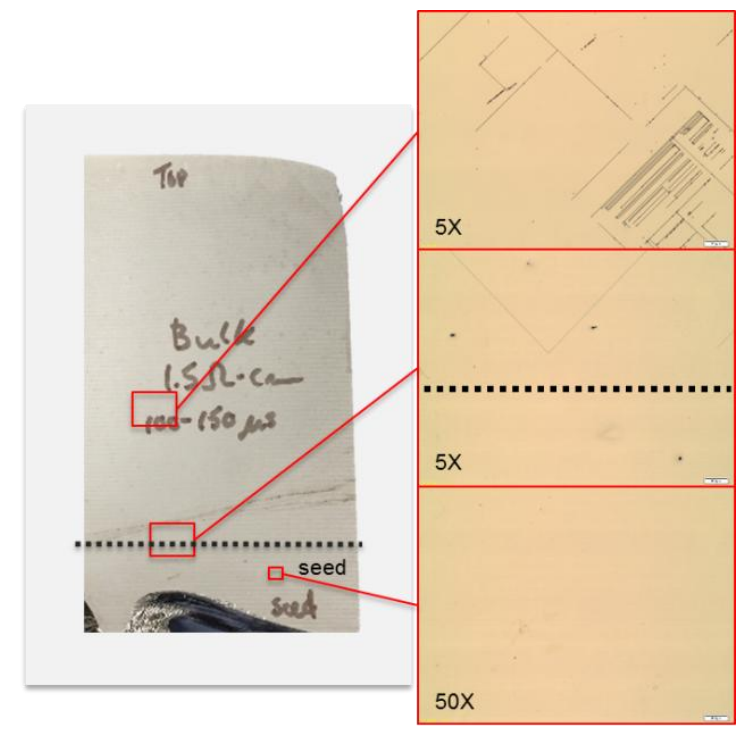

Fig. 5: Photo of a section from Ingot 1 and preferential defect etch from different areas.

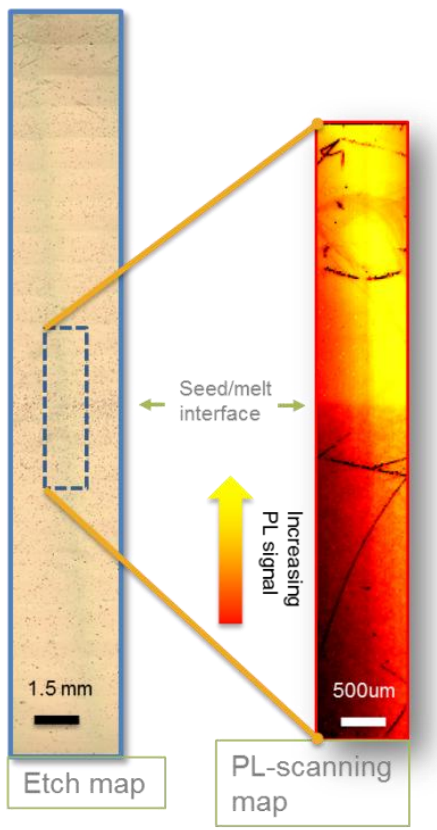

Fig. 6: Defect etch map and Photo-luminescence map from Ingot 2 


\section{Citations}

${ }^{1}$ W. C. Dash, J. Appl. Phys. 30 (1959), p. 459

${ }^{2}$ H. Siethoff, from Properties of Crystalline Silicon, R. Hull, ed. (1999), p. 123

${ }^{3}$ E. Takasuka et al., J. Appl. Phys. 81, (1997), p. 6384

${ }^{4}$ S C Jain et al., J. Phys. D: Appl. Phys. 4 (1971), p. 1207

${ }^{5}$ C. Donolato, J. Appl. Phys. 84 (1998), p. 5

${ }^{6}$ N. Stoddard, B. Wu, I. Witting, M. C. Wagener, Y. Park, G. A. Rozgonyi, R. Clark, Solid State Phenomena, 131-133, (2007), pp. 1-8

${ }^{7}$ Y. Miyamura, H. Harada, K. Jiptner, J. Chen, R.R. Prakash, S. Nakano, B. Gao, K. Kakimoto, T. Sekiguchi, J. Crystal Growth 401 (2014) p. 133

${ }^{8}$ K. Nakajima, R. Murai, S. Ono, K. Morishita, M. Kivambe, D. M. Powell, and T. Buonassisi, Japanese Journal of Applied Physics, 54(1), (2015), p. 015504.

${ }_{9}^{9}$ M.Trempa, C. Reimann, J. Friedrich, G. Müller, A. Krause, L. Sylla, T. Richter, J. Crystal Growth 405 (2014) p. 131

${ }^{10}$ M. Trempa, C. Reimann, J. Friedrich, G. Müller, L. Sylla, A. Krause, T. Richter, J. Crystal Growth 429 (2015) p. 56 
- 